\title{
Estudio tipológico de los observatorios astronómicos
}

\section{A typological study of astronomical observatories}

\author{
Miguel Ángel Castro-Tirado ${ }^{(*)}$, Alberto Javier Castro-Tirado $^{(* *)}$
}

\section{RESUMEN}

El desarrollo de la astronomía como ciencia, de los sistemas constructivos y de la tecnología ha provocado una evolución no planificada de los observatorios astronómicos como edificios. Estos cambios se han consolidado mediante un proceso de ensayoerror en el que determinadas innovaciones se iban descartando mientras algunas novedades se consolidaban y otras modificaciones sustituían soluciones preexistentes. Como es lógico, a lo largo de este proceso también se han producido algunos proyectos fallidos o parcialmente errados. A tenor de la constante evolución de los parámetros que condicionan las necesidades y posibilidades técnicas de los observatorios, este trabajo clasifica estos centros astronómicos, proponiendo 10 tipologías en el desarrollo de los observatorios astronómicos a lo largo de la historia, y estudia los rasgos que los definen para establecer un conocimiento de base que sirva a los diseñadores de futuros proyectos.

Palabras clave: Observatorios astronómicos, tipología, tipos arquitectónicos, desarrollo del observatorio.

\section{ABSTRACT}

The development of astronomy as a science, construction systems and technology has led to an unplanned evolution of astronomical observatories such as buildings. These changes have been consolidated through a process of trial and error in which certain innovations were discarded while some new features were consolidated and other modifications replaced pre-existing solutions. As is logical, during this process there have also been some failed or partially erroneous projects. According to the constant evolution of the parameters that determine the needs and technical possibilities of the observatories, this paper classifies these astronomical centers, proposing 10 types in the development of astronomical observatories throughout history, and study the features that define them to establish a basic knowledge that will serve the designers of future projects.

Keywords: Astronomical observatories, typology, architectural types, observatory's development.

(*) Doctor Arquitecto profesor de la Escuela de Arquitectura, Universidad de Málaga (España).

(**) Astrofísico. Profesor de Investigación del Instituto de Astrofísica de Andalucía del Consejo Superior de Investigaciones Científicas (IAA-CSIC). Granada (España).

Persona de contacto/Corresponding author: ajct@iaa.es (A.J. Castro-Tirado).

ORCID: https://orcid.org/oooo-0002-0841-0026 (M.A. Castro-Tirado); https://orcid.org/oooo-0oo3-2999-3563 (A.J. Castro-Tirado).

Cómo citar este artículo/Citation: Castro-Tirado, Miguel Ángel; Castro-Tirado, Alberto Javier (2020). Estudio tipológico de los observatorios astronómicos. Informes de la Construcción, 72(559): e348. https://doi.org/10.3989/ic.72890.

Copyright: (C) 2020 CSIC. Este es un artículo de acceso abierto distribuido bajo los términos de la licencia de uso y distribución Creative Commons Reconocimiento 4.0 Internacional (CC BY 4.0). 


\section{INTRODUCCIÓN}

El observatorio astronómico tiene sus primeros antecedentes en estructuras o arcaicas construcciones asociadas con el registro de diversos fenómenos o eventos celestes. Estos proto-observatorios o puestos de observación se remontan varios milenios en el tiempo con ejemplo como los conjuntos del Círculo de Goseck, Nabta Playa, Mnajdra o Stonhenge (1). Todos esos precedentes pertenecen a civilizaciones primitivas con un bajo grado de tecnología e incluso, habitualmente, hábitos nómadas, lo que favorece una relativamente simple identificación de sus construcciones. En este sentido, la evolución hacia culturas más complejas (Egipto, Mesopotamia, China, Grecia o Roma) dificulta esta tarea ya que las civilizaciones más avanzadas tienden a crear asentamientos de múltiples edificios que no alcanzan un grado preciso de especialización que requiere la observación (2). Este reconocimiento del cielo podría ser llevado a cabo a simple vista desde cualquier construcción o, incluso, en los alrededores de éstas a cielo abierto. Aunque se pueden encontrar referencias al uso como observatorio de la Torre de Babel o a la presencia de algún tipo de institución astronómica dirigida por Tolomeo en Alejandría (3, 4), no hay evidencia que respalde la existencia de un observatorio astronómico pre-medieval.

Durante la Edad Media, la cultura islámica ocupa un papel esencial en la historia de la astronomía, preservando el vasto conocimiento griego sobre esta materia e incorporando sus propios hallazgos y aportaciones. Todo ello sería recuperado para occidente por la Escuela de Traductores de Toledo de Alfonso X durante el siglo XIII (5). El observatorio islámico-medieval, como institución, solo será posible como lógica consecuencia del desarrollo del legado griego. El observatorio, como tal, aparece por primera vez en esta civilización en tanto que los rasgos de la cultura islámica como sociedad enlazan con el observatorio como institución científica (6).

Aunque algunas referencias atribuyen el primer observatorio europeo a Regiomontanus o a Copérnico $(7,8)$, no hay ninguna evidencia de una construcción o de instrumentos fijos que sostengan estas afirmaciones (9). Por otro lado, hay un gran consenso al situar en el palacio del Wilhelm IV de Hesse-Kassel el primer observatorio moderno $(10,11)$. No obstante, una plataforma construida a modo de mirador en el palacio a donde sacar los instrumentos portátiles (12) no representa un avance desde la perspectiva de la arquitectura. Esto no invalida su importancia ya que será una visita a estas instalaciones lo que inspire a Tycho Brahe para, unos meses más tarde, construir en Hven los primeros observatorios modernos, Uraniborg (1576) y Stjerneborg (1584) (13), que se caracterizarán por contar con cierto grado de intencionalidad y especialización arquitectónica para la astronomía.

La aparición del telescopio a principios del siglo XVII y su pronta vinculación con la astronomía iba a condicionar el devenir del diseño de los observatorios. Sin embargo, dado que los primeros telescopios solían ser de un tamaño reducido y bastante manejables, numerosos astrónomos llegaron a la conclusión de que podrían realizar sus observaciones desde cualquier lugar. Es por esto que, durante este periodo, el desarrollo de los centros astronómicos tiende hacia estructuras sencillas o, directamente, a añadidos sobre edificaciones preexistentes para instalar plataformas desde las que conseguir mejores visuales del cielo, como en las universidades de Leiden (1633) e Ingolstadt (1635) o en el Rundetaarn danés (1637) (14). Por lo tanto, pasarían muchos años entre la aparición del primer observatorio moderno y la consolidación de la idea de que un centro destinado a la observación científica requería de un edificio concebido expresamente para dicha finalidad.

La fundación del Observatorio de París (1667) marca la primera construcción de un nuevo edificio para una finalidad astronómica. El edificio resultante es un palacio donde la función institucional comparte espacio con las residencias y las zonas dedicadas a la astronomía: salas de observación, un cuarto de estudio cenital en el sótano y una cubierta plana transitable para disponer instrumentos $(15,16)$. Durante más de un siglo, los observatorios desarrollados siguiendo el ejemplo del francés, se limitaron a proponer, con sus pequeños matices, edificios más o menos artificiosos en los que aunaban residencia, zona de trabajo y unos espacios donde poder operar los telescopios. Así se reprodujeron las altas salas de observación con ventanales verticales como en el Observatorio de Greenwich (1675) (17) o se implantaron las aperturas de norte a sur a partir del Observatorio Radcliffe (1772) (18).

El Observatorio de Dunsink (1785) incorporó importantes cambios que serían replicados por los posteriores centros astronómicos. En este caso, su localización se determinó en base a las condiciones del lugar, el edificio tuvo en consideración la estabilidad termal y estructural, y el diseño reservó al telescopio una posición privilegiada (19). Esta tendencia continuó durante los siguientes doscientos años mientras los nuevos observatorios incluían nuevos usos y los telescopios se hicieron cada vez más grandes.

El desarrollo de las ciudades a partir del siglo XIX empieza a propiciar problemas ya en algunos observatorios decimonónicos, que no solo se comienzan a retirar de las grandes capitales hacia poblaciones menores sino que empiezan a ver como algo favorable su situación en entornos alejados de lo urbano. Además, la búsqueda de la máxima precisión posible de los instrumentos devendrá en la optimización las condiciones de observación, lo que conllevará el establecimiento de estas instituciones en zonas, en la medida de lo posible, no afectadas por la contaminación antrópica y con las condiciones atmosféricas más favorables a su disposición. Esto supondrá su aislamiento de los centros urbanos, en zonas remotas y elevadas como en cimas de montañas, lejos de los humos y luces de la ciudad. Como contrapartida esto implicará dificultades en los suministros públicos (electricidad, agua...) y en las comunicaciones.

En esta línea, el Observatorio Lick (1881) se iba a convertir en el primer observatorio de montaña en el mundo ocupado de manera continua $(20,21)$. Sin embargo, las características arquitectónicas del edificio eran más propias del observatorio moderno que del contemporáneo. Eso es por lo que el Observatorio Lick representa un caso intermedio, de transición entre ambas corrientes. Aun así, casi simultáneamente, el Observatorio de Niza (1881) surgiría como un conjunto de estructuras independientes distribuidas sobre la cumbre de una montaña. Cada diferente edificio se concibió específicamente para una función concreta que justificaba su diseño y posición. Incluso incorporó las primeras construcciones des- 
tinadas íntegramente a un telescopio. De este modo, se convertiría en el precursor de la configuración del observatorio contemporáneo como un conjunto de edificios independientes y especializados (22).

Desde este momento, será el crecimiento del telescopio lo que condicione el diseño del observatorio. Su aumento de tamaño producirá un incremento en la escala de la construcción que permitiría la reintroducción de algunos usos en la base del edificio. Tras el desarrollo inicial del telescopio Hooker (1917), el telescopio Hale (1948) terminó por consolidar la inclusión de diferentes funciones en su base y llegando a acoger en su inauguración a casi mil personas bajo su cúpula $(23,24)$.

A partir de los avances en informática introducidos por primera vez en un gran observatorio en el BTA-6, la relación entre el telescopio y sus usuarios se podía realizar a través la sala de control. Esto permitía aislar al instrumento principal de observación de otras funciones del observatorio que podían perjudicar las condiciones de investigación (25). La posibilidad de dividir el observatorio en dos estructuras independientes, una para el telescopio y otra para el resto del programa de usos, se vería reforzada gracias al progreso en telecomunicaciones que, aplicado por primera vez en el W. M. Keck, permitiría realizar las observaciones desde cualquier ordenador del mundo (26).

Hoy en día, aunque no es necesario que un astrónomo "mire a través de una lente" (como en los viejos tiempos) para acceder a una observación, todavía hay una serie de tareas derivadas de ésta que requieren un espacio en el que los científicos e ingenieros puedan trabajar. Un estudio sobre la tipología arquitectónica de los observatorios puede ser una forma adecuada de reflexionar sobre cómo debería ser el próximo observatorio astronómico y cómo diseñar el edificio que mejor se adapte a las necesidades de su organización bipolar.

\section{SOBRE LA TIPOLOGÍA ARQUITECTÓNICA}

Dejando a un lado determinadas construcciones en serie propias de los grandes desarrollos urbanos producidos a partir del siglo XX, las obras de arquitectura son, en general, productos singulares ya que se caracterizan por multitud de parámetros (definición formal, funcionamiento espacial, programa de usos, solución constructiva...) que las diferencian. Teniendo en cuenta su desarrollo histórico, los observatorios astronómicos, por su propia infrecuencia, no pertenecen a esas producciones seriadas y repetitivas.

Aun así, en toda obra se pueden identificar algunos rasgos generales o patrones a partir de los que establecer clasificaciones, sin que ello tenga porqué poner en cuestión su individualidad.

El mero hecho de nombrar, de atribuir un nombre, a un objeto arquitectónico constituye un acto que, por la naturaleza misma del lenguaje, fuerza a la tipificación. Es decir, el lenguaje reconoce implícitamente el concepto de tipo, puesto que, fundamentalmente, se basa en la posibilidad de agrupar objetos a partir de ciertas similitudes estructurales que le son inherentes (27). A través de este proceso de pensamiento semántico la arquitectura se conceptualiza en categorías que relacionan y clasifican diferentes construcciones o elementos arquitectónicos en función de algún atributo común.

La propia ordenación conceptual en conjuntos implica la existencia de subtipos a medida que se concretan las reglas de agrupación (por ejemplo el subtipo "mamífero" pertenece al tipo "animales" ya que todos los mamíferos son animales) y posibilita que un mismo elemento pueda pertenecer a diferentes conjuntos independientes en función de los criterios de selección considerados (por ejemplo los tipos "mamífero" y "cuadrúpedo" ya que se puede pertenecer a ambos tipos, pero también solo al primero o solo al segundo).

Algunas acepciones del Diccionario de la Lengua Española de la Real Academia Española son bastante aclaratorias en este sentido, ya que definen al tipo como "símbolo representativo de algo figurado", como "clase, índole o naturaleza de las cosas", y como "ejemplo característico de una especie, de un género". Sin embargo, su primera acepción es "modelo", que según la misma fuente es un "punto de referencia para imitarlo o reproducirlo", lo cual es contrario al significado de tipo en la arquitectura.

Precisamente sobre esa importante diferencia entre "tipo" y "modelo" teorizó el filósofo y crítico de arte Quatremère de Quincy en su Dictionnaire historique de l'architecture. El origen de la palabra "tipo" proviene del griego typos, que significa modelo, matriz, impresión, molde, etcétera. Sin embargo, en la actualidad existe un contraste importante entre los significados de ambas palabras. El modelo es una imagen precisa a copiar, un objeto particular y absolutamente definido que puede ser replicado tal cual es. El tipo, por el contrario, es un concepto menos concreto y más abstracto que constituye la idea que puede servir de regla a una infinidad de posibles modelos distintos, con variantes, pero siempre en base a un esquema general y con una esencia común (28).

El tipo arquitectónico no surge de manera espontánea, sino que aparece como noción de ciertas formas generales y características de una construcción a partir del desarrollo en el tiempo de un motivo y un trasfondo histórico-cultural. La protección y la generación de un espacio abierto semiprivado subyace tras el patio de la domus romana, como lo hace en la corrala o en el espacio interior de los edificios en manzana cerrada. Sin embargo, ese proceso no responde necesariamente a una evolución lineal. Esto es, un mismo tipo puede desarrollarse de forma divergente en diferentes términos y dos familias distintas pueden converger; incluso algunas circunstancias pueden producir la exinción de un tipo de igual modo que determinadas necesidades puedan provocar la aparición de uno nuevo. En todo caso, estos procesos son el resultado de un proceso orgánico, difícilmente alcanzable desde las imposiciones ideológicas.

Quatremère de Quincy ya advertía que tanto los que pretenden liberar la arquitectura de toda regla para someterla a su capricho, como los que la encadenaban a la estricta imitación desnaturalizaban la arquitectura. Ésta se sostiene en la identidad y singularidad propia de un proyecto, en unas circunstancias particulares, adaptado a un esquema general y a unas trazas que el paso del tiempo ha consolidado (28).

Precisamente los teóricos que a principios de siglo XX pretendían renovar la arquitectura se opusieron al concepto del tipo decimonónico ya que lo entendían como inmovilismo, 
como restricciones a un creador que debía tener total libertad. Sin embargo, esa nueva arquitectura de producción en masa comenzaría a cuestionar la singularidad del objeto arquitectónico (29). El abandono de los tipos históricos por una vocación funcionalista (relacionando directamente función y forma como causa y efecto) no hacía sino ofrecer unas nuevas reglas que articularían, con el tiempo, nuevos tipos arquitectónicos.

Como contraposición a la concepción apriorística de Quatremère según la cual el tipo era un ideal más o menos arbitrario, el historiador y crítico de arte Argan define el tipo como una deducción a posteriori a partir de la comparación de una serie de edificios, idealmente de todos, para identificar los rasgos semejantes o asimilables para un determinado conjunto de proyectos a los que denominar de una forma particular (30)

Al definir la tipología, Argan parte de una arquitectura a la que se le presupone que la forma tiene relación y continuidad con la función. A partir de esto, considera los aspectos formales de una serie de elementos arquitectónicos en base a una función común o a una imitación, de lo que se deduce cierta antinomia entre tipología y creatividad (31).

Asimismo, Argan plantea que la tipología, entendida como el estudio y la clasificación de los tipos, puede considerar los tipos arquitectónicos en función de diferentes criterios. Así presenta una tipología referida a la configuración espacial del edificio, donde podría hablarse de construcción extensiva o en altura, edificio compacto o conjunto disgregado, espacio diáfano o compartimentado... Por otro lado identifica un tipo en relación a la función para la que se diseña el edificio, en el que se encontrarían el colegio, la vivienda, la iglesia, el teatro, el hospital...Por último alude a una tipología de elementos del edificio en la que encajarían la columna, la cúpula, el forjado, el cerramiento, la ventana... (30). De cualquier modo, esas clasificaciones son solo algunas de las ilimitadas categorías a partir de las que se podrían definir tipos de arquitectura. Así, por ejemplo, se puede ordenar la arquitectura en función del tipo de estructura, del tipo de envolvente, del tipo de acabados, del tipo de permeabilidad pública, del tipo de integración en su contexto, del tipo de situación respecto de la ciudad, del tipo de estrategia climática, etcétera.

Independientemente de la proposición ideal que sustentó Quatremère de Quincy o de la deducción estructural que propuso Argan, el tipo en arquitectura responde a un ejercicio de abstracción intelectual que define conceptos con los que poder agrupar objetos arquitectónicos.

El análisis de un determinado tipo arquitectónico con una visión retrospectiva muestra como el tipo no define un modelo invariable sino que se transforma y autorregula. Presenta variaciones más o menos sutiles que en el transcurso del tiempo pueden traducirse en cambios significativos. Por todo ello el tipo en arquitectura funciona como una estructura genética para un determinado conjunto de edificios (31). Incluso ciertos sucesos o eventos pueden incidir de manera sustancial sobre su esencia. Los tipos se transforman entonces dando lugar a otros nuevos cuando los elementos substanciales de su estructura cambian (27).

El tipo se constituye mediante un proceso de selección en el que se descartan aspectos particulares de un conjunto y se identifican aquellas características coincidentes y constantes en todos los casos. A partir de esa criba, se conceptualiza el esquema de rasgos como la definición de una idea (30).

De este modo, el tipo permite identificar la arquitectura a partir del reconocimiento de determinados atributos, ayuda a comprender la relevancia de ciertas tendencias arquitectónicas (soluciones constructivas, presencia de elementos, organizaciones espaciales...) en el tiempo, posibilita un conocimiento de base para el proceso creativo y favorece cierto juicio ante potenciales proyecto de ese tipo u otros semejantes (32).

En cualquier caso, el tipo es un constructo intelectual que racionaliza y simplifica una realidad compleja para generar una herramienta que permite analizar y comprender el pasado de la arquitectura, y establecer unos criterios a partir de los que proyectar edificios en el futuro.

En este último sentido, a la hora de afrontar un nuevo proyecto, el arquitecto debe identificar el tipo arquitectónico sobre el que va a trabajar. Sin embargo, esto no debe traducirse en un ejercicio de imitación o repetición literal. Es decir, para trabajar con un determinado tipo de edificación, se estudian los edificios de ese tipo (teóricamente todos) y se establece un desarrollo conceptual del mismo como punto de partida. El arquitecto trabaja sobre ese concepto mediante transformaciones, transgresiones, desde su simplificación o a través del respeto espacio-constructivo. Sin embargo, toda actuación parte del reconocimiento del tipo. A través del desarrollo definitorio de un determinado tipo, que no es más que una idea sintética e indefinida, el concepto abstracto original se va concretando en un proceso guiado por las decisiones del arquitecto hasta una solución singular $(29,30)$.

\section{EL OBSERVATORIO ASTRONÓMICO COMO UN NUEVO TIPO}

La naturaleza y la razón de ser en el presente del propio tipo arquitectónico son puestas en cuestión por Moneo al reflexionar sobre si la mera concepción del concepto tipo, si la conciencia de su noción, no quebranta la unidad evolutiva que lo fundamenta; o si el reconocimiento de esos desarrollos no supone una señal de su caducidad. En la transformación sufrida por la arquitectura desde principios de siglo $\mathrm{XX}$, con cambios técnicos, sociales y teóricos, radica la complejidad para aplicar el concepto del tipo a la arquitectura actual. Sin embargo, el hecho de que el fruto de la arquitectura no constituya, en general, un hecho aislado, singular e irrepetible evidencia la pertinencia de los tipos. Si reconociendo la individualidad de una obra se pueden identificar patrones o características que permiten su clasificación y agrupación con otros objetos arquitectónicos, es una obviedad que una interpretación tipológica adecuada a los nuevos procesos proyectivo-constructivos es posible. Es decir, que el concepto de tipo es intrínseco a la propia naturaleza de la producción arquitectónica (27).

Entonces, la innovación técnica o la aparición de una nueva necesidad traducida en una función programática novedosa puede requerir de una solución proyectual que no encaje en ningún tipo existente hasta ese momento. Partiendo de la base tipológica que justifica cada tipo como una evolución de tipos pasados constituidos a partir de la abstracción de los atributos comunes a un conjunto de elementos arquitectónicos, ¿cómo surge ese nuevo tipo? 
Argan apunta la imposibilidad de crear un nuevo tipo arquitectónico, justificando que siempre es fruto de una deducción a partir de experiencias históricas (30). Moneo, por su parte, acepta la premisa de la creación de un nuevo tipo como algo natural y habitual en el desarrollo en el tiempo de la historia. Y aun reconociendo la dificultad que esto supone, apunta hacia los tipos -otros tipos- como punto de partida del diseño (27). Por último, Quatremère de Quincy sustenta que los edificios deberían encontrar en su propósito fundamental, en su función o usos, un tipo que sea conveniente para ello (28). De manera que alude a la capacidad de adaptación o mutabilidad de los tipos para acomodarse a nuevas necesidades.

Así que la mera función investigadora astronómica podría constituir un criterio de agrupación para los observatorios, aunque resultaría una clasificación vaga y poco precisa ya que hay otros aspectos relevantes que condicionan la naturaleza de su construcción. En este sentido, se puede considerar el observatorio astronómico como un tipo de edificio basado en su actividad investigadora astronómica pero, ¿̇es posible establecer unas categorías tipológicas sobre la arquitectura de los observatorios astronómicos?

Müller, el primer autor en definir los tipos de observatorios, establece una clasificación (Figura 1) basada en características formales y, sustancialmente, en la composición de las plantas. Delimita nueve categorías: en forma de grupo, en forma de línea, en forma de L, en forma de U, en forma de $\mathrm{H}$, en forma de cruz, en forma de $\mathrm{T}$, en forma triangular $\mathrm{y}$ en forma de torre (33). Esta ordenación divide en exceso los edificios en función de la distribución de sus alas, definiendo varios conjuntos con rasgos muy similares y fácilmente asimilables. Además, podría ser pertinente para los observatorios del siglo XIX, pero no permite organizar otros anteriores, como el de Greenwich, o posteriores, como el BTA-6.

Más recientemente, Waumans cataloga lo que denomina como los subtipos de observatorio. En este caso, considerando tanto aspectos formales como funcionales, presenta una clasificación (Figura 2) con seis conjuntos: torre, cúpula centrada, cúpulas descentradas, división total, funciones en la cúpula y división frío-calor (34). Incluso valorando como positivo su mayor grado de definición y una menor arbitrariedad clasificatoria que el precedente de Müller, algunos observatorios no encajan en estos tipos, aun cuando no constituyen una excepción, (como el

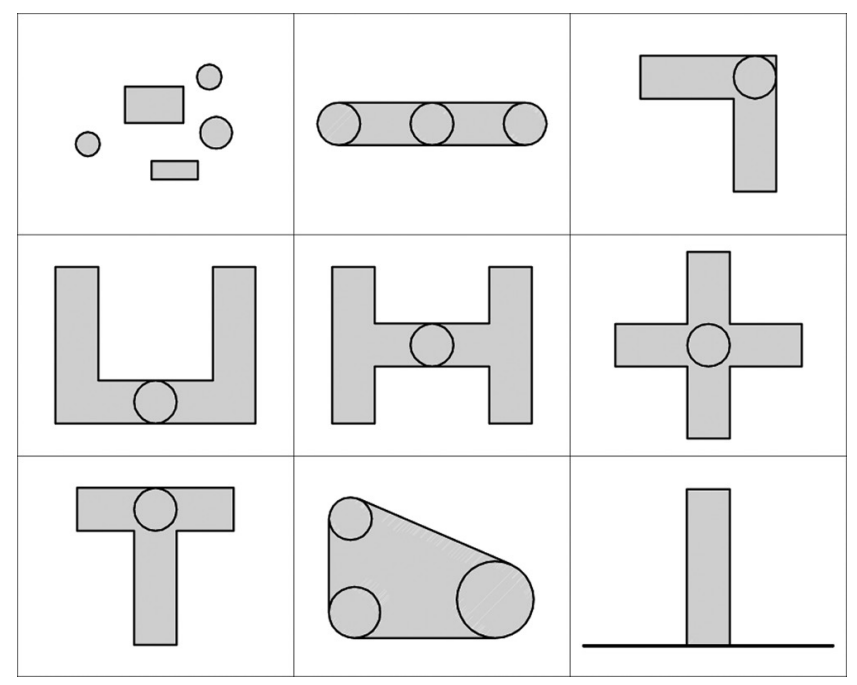

Figura 1. Diagrama de tipos propuestos por Müller.

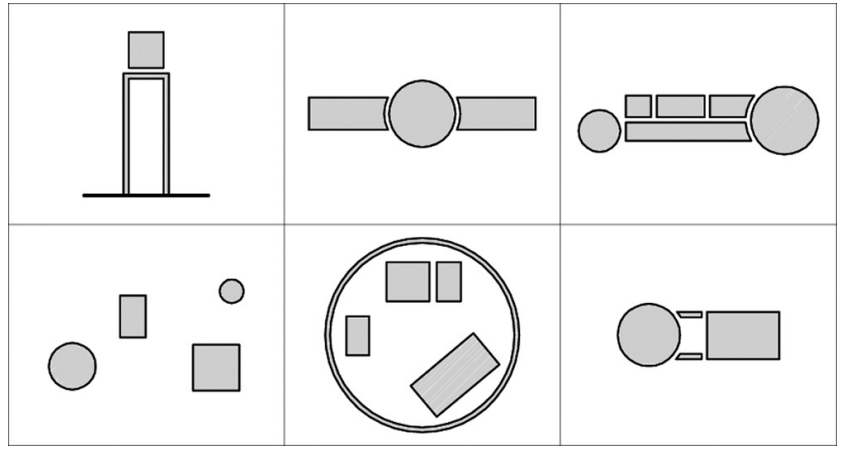

Figura 2. Diagrama de tipos propuestos por Waumans.

Radcliffe o el de Madrid...), el encaje de otros es confuso (como el primer observatorio de Hamburgo), y otros ni siquiera son contemplados en el estudio (los islámico-medievales) ya que se centra en los casos posteriores a la recuperación occidental de la astronomía durante el siglo XIII.

\section{PROPUESTA DE 10 TIPOS DE OBSERVATORIOS ASTRONÓMICOS}

Por todo lo expuesto, se presenta aquí una clasificación tipológica que, partiendo de las anteriores, recoja los proyectos obviados e, incluso, integre alguna solución astronómica que sin constituir un observatorio en sí mismo, forme parte de la evolución histórica de éstos. De cualquier modo se asume la existencia de algunos casos que por su excepcionalidad o singularidad se escapan a esta categorización (como Stjerneborg o el McMath-Pierce). Los tipos identificados en el desarrollo de esta institución astronómica son: de arco, plataforma, palaciego, de sala de observación, de torre, de cúpula integrada, de cúpula sobredimensionada, disgregado, compacto en cúpula y de cúpula con anexo.

El tipo de arco (Figura 3) se refiere a los observatorios del periodo islámico-medieval. Éstos se distinguen por la presencia de un gran instrumento de arco graduado, sextante o cuadrante, que ocupa el espacio central de la construcción en toda su altura. El edificio en el que se integra es de planta circular, quedando el espacio restante destinado a otros usos vinculados con la actividad astronómica como la biblioteca, las salas de cálculo o de almacenaje de instrumentos portátiles. Por ejemplo: el de Maragha o el de Samarcanda.

El tipo plataforma (Figura 4) no alude a una clase de observatorio propiamente dicho, sino que agrupa los puestos de observación característicos en la reintegración de la astronomía en Europa y a los que posteriormente surgirían con la aparición de los primeros telescopios con fines astronómicos. Este tipo se estructura como una superficie abierta o, en ocasiones, con

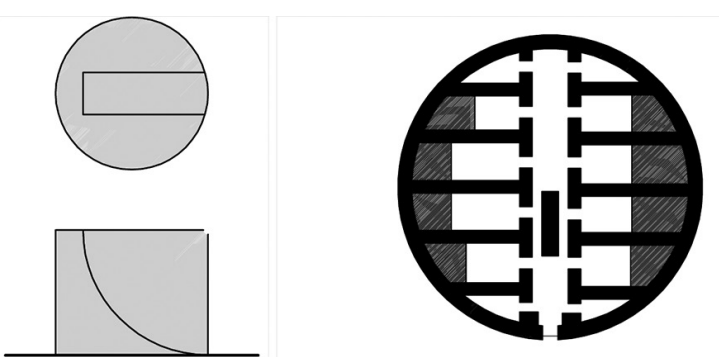

Figura 3. Esquema del tipo de arco. Planta del Observatorio de Maragha como ejemplo. 
una habitación cubierta, que frecuentemente se adosa sobre edificaciones existentes para conseguir unas mejores condiciones visuales del entorno y desde donde realizar estudios astronómicos con instrumentos portátiles. Por ejemplo: el puesto de observación de Leiden o la plataforma de Hevel.

El tipo palaciego (Figura 5), como su nombre evidencia, señala la adaptación de un tipo existente, de carácter residencial o institucional, al uso como observatorio. En este caso, las plantas, secciones y alzados del proyecto difícilmente evidencian rastro alguno del uso astronómico para el que se acondicionan, existiendo solo algunos detalles que, para un observador versado, apuntan esa finalidad. El edificio incluye ya, además de usos complementarios a la observación astronómica, un espacio residencial. Por ejemplo: el de París o el de Cádiz.

El tipo de sala de observación (Figura 6) apunta a aquellos observatorios en los que, más allá de otros espacios de trabajo o de usos secundarios, existe una cámara adaptada a la observación con telescopios portátiles. Ésta, además de contar con altos huecos en fachada apuntando en todas direcciones, se reconoce por su posición elevada y dominante sobre el conjunto para favorecer la visión libre de obstáculos. Por ejemplo: el Observatorio de Greenwich y el Radcliffe.

El tipo de torre (Figura 7) se configura en base a una esbelta construcción en altura cuya justificación, a pesar de su perjuicio a la estabilidad estructural, radica en su situación en un entorno urbano o periurbano, por lo que la presencia de edificaciones y obstáculos en su entorno hacen necesaria una elevación del

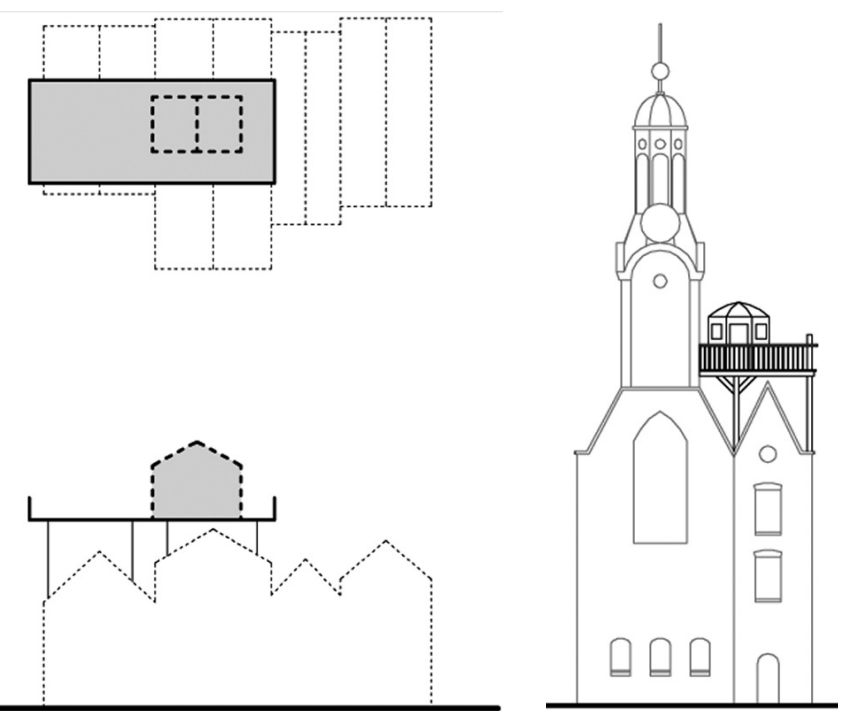

Figura 4. Esquema del tipo plataforma. Alzado de la Plataforma de observación de Leiden como ejemplo.
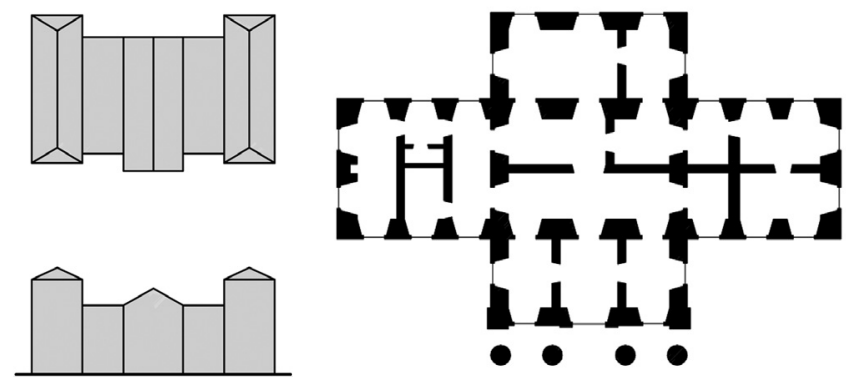

Figura 5. Esquema del tipo palaciego. Planta del Observatorio de Cádiz como ejemplo. observatorio para acceder a una visual del cielo. Independientemente de los otros usos de las plantas inferiores, el nivel superior suele estar destinado a los principales instrumentos del complejo. Aunque su origen está en la recuperación occidental de la astronomía, su uso en instituciones docentes o divulgativas de la ciudad ha prolongado su existencia hasta la actualidad. Por ejemplo: el Observatorio de Mannheim y el de Bogotá

El tipo de cúpula integrada (Figura 8) aparece con la evolución de la sala de observaciones a un espacio especializado donde el telescopio ocupa el papel principal: queda instalado en un soporte fijo bajo una cúpula móvil y con un hueco practicable que permite observar todo el cielo manteniendo el instrumento a resguardo. Con una disposición horizontal del edificio donde se distribuye el resto del programa, las cúpulas serán el elemento que destaque por encima del resto del observatorio. Por ejemplo: el de Dunsink o el de Göttingen.

El tipo de cúpula sobredimensionada (Figura 9) se caracteriza por el crecimiento de una cúpula a causa del aumento de tamaño del instrumento principal. Esto provoca una descompensación entre los volúmenes de la construcción que provocan el desplazamiento de esta cúpula a un extremo de la planta para evitar su interferencia con otras funciones del observatorio o minimizar la obstrucción de otros instrumentos. Por ejemplo: el Observatorio Lick o el Yerkes.

El tipo disgregado (Figura 10) aparece como respuesta a los problemas que la coincidencia de usos tan dispares como la residencia y la observación en un mismo edificio producen. De ahí que el observatorio se escinda en un grupo de construcciones independientes cada una de las cuales está especialmente concebida para su propia finalidad. Incluso cada instrumento astronómico cuenta con su propio edificio adap-

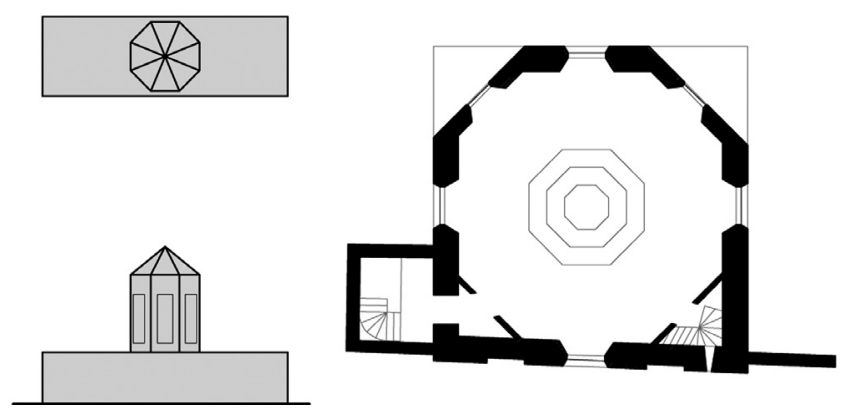

Figura 6. Esquema del tipo sala de observación. Planta del Observatorio de Greenwich como ejemplo.

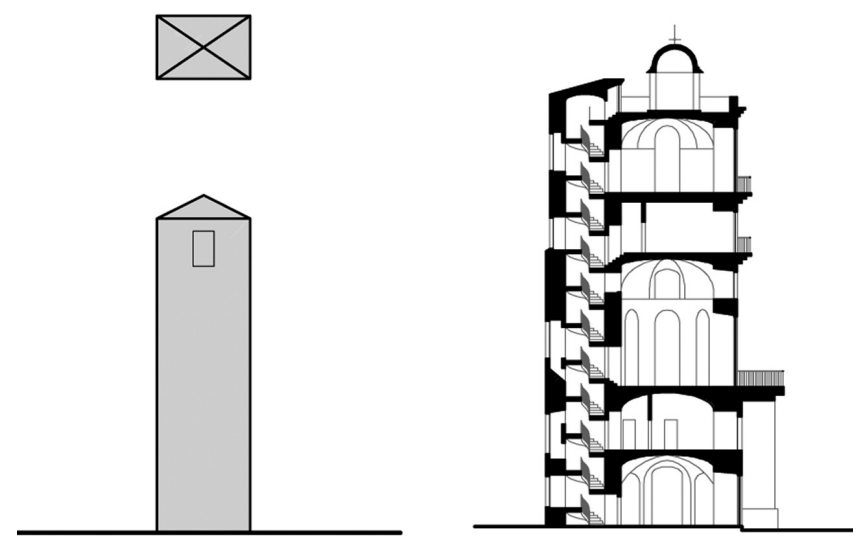

Figura 7. Esquema del tipo de torre. Sección del Observatorio de Mannheim como ejemplo. 


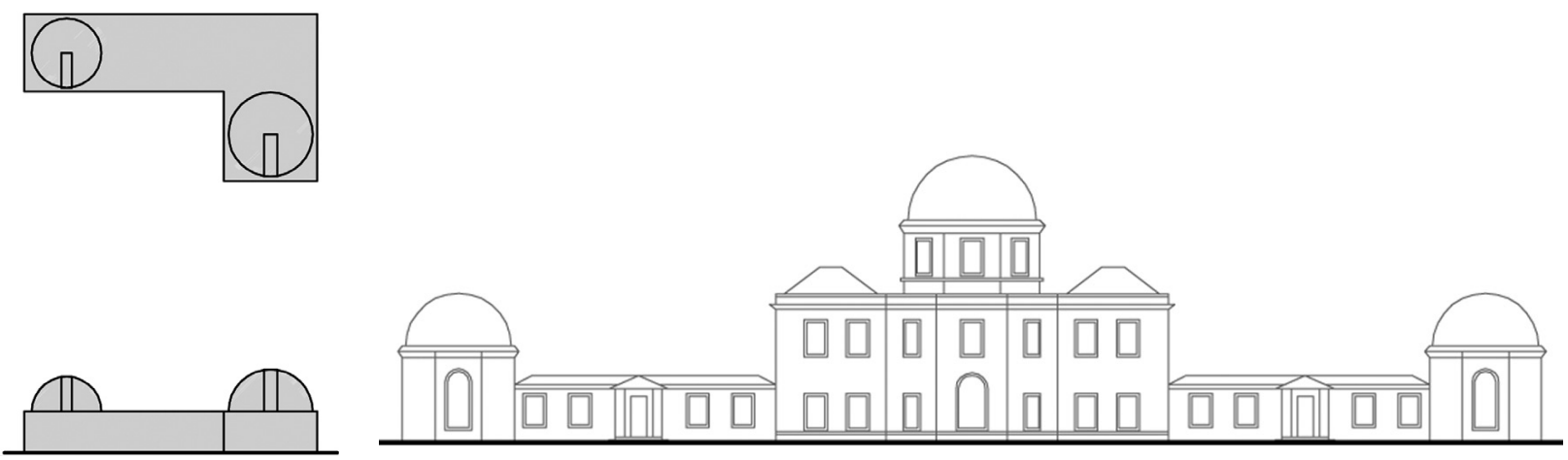

Figura 8. Esquema del tipo de cúpula integrada. Alzado del Observatorio de Dunsink como ejemplo.

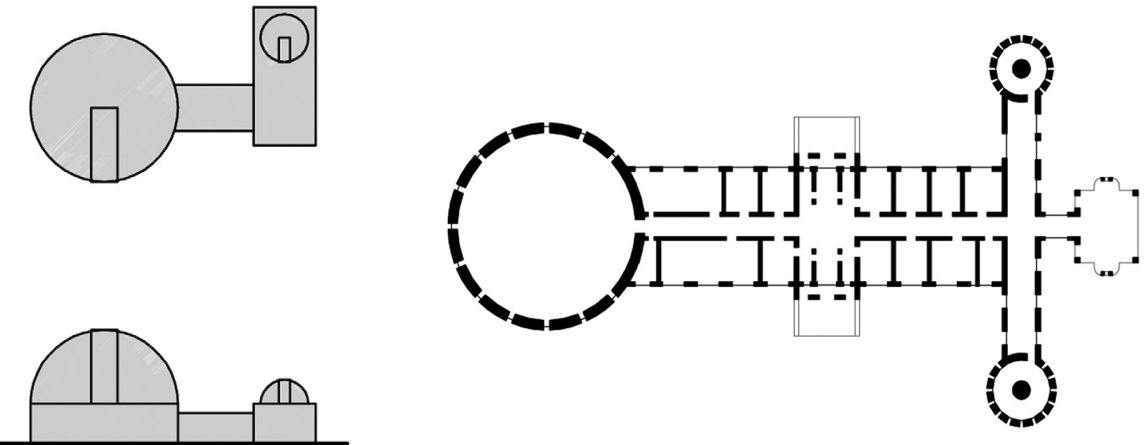

Figura 9. Esquema del tipo de cúpula sobredimensionada. Planta del Observatorio Yerkes como ejemplo.

tado expresamente a sus necesidades. Por ejemplo: el Observatorio de Niza o el Mount Wilson.

El tipo compacto en cúpula (Figura 11) es la consecuencia lógica del aumento de escala de los telescopios y, por ende, de la edificación que lo alberga. Los nuevos reflectores alcanzan unas dimensiones tales que la planta de la cúpula que los cubre es tan grande que la superficie que encierran permite insertar en su interior todo el programa de usos propio de un observatorio. De forma que la mayor parte de las funciones que se situaban diseminadas por un terreno en el tipo disgregado se apilan en la base del telescopio principal en esta variante. Por ejemplo: el Hale o el BTA-6.

El tipo de cúpula con anexo (Figura 12) se aplica a los observatorios en los que el control remoto de los equipos astronómicos permite la separación física de la cúpula donde está el telescopio, con un segundo volumen donde se encuentran la sala de control y otros espacios de trabajo o servicio para los usuarios del centro. Es decir, mientras que la cúpula se adecua a los requisitos del reflector, el anexo se adapta al confort y las características del resto de usos. La cúpula y el anexo funcionan como dos edificios independientes cuya verdadera conexión, la que los unifica como una sola institución, se produce a través de cables o de ondas electromagnéticas, por lo que este vínculo es, en realidad, virtual y podría llegar a ser equivalente si la aumentase la distancia entre ambas partes. Por ejemplo: el Observatorio W. M. Keck y el Gran Telescopio Canarias.

Mientras que el tipo de arco se limita al periodo islámico medieval, el resto de tipos se suceden y superponen a partir de la reintegración de la astronomía en Europa. El tipo plataforma aparece con los primeros precedentes occidentales del observatorio y se solapará con el palaciego hasta que el telescopio adquiera suficiente relevancia como para que el tipo de sala de observación y el de torre se implanten. El aumento en importancia del telescopio propiciará el desarrollo del tipo de cúpula integrada, que reemplazará al de sala de observación
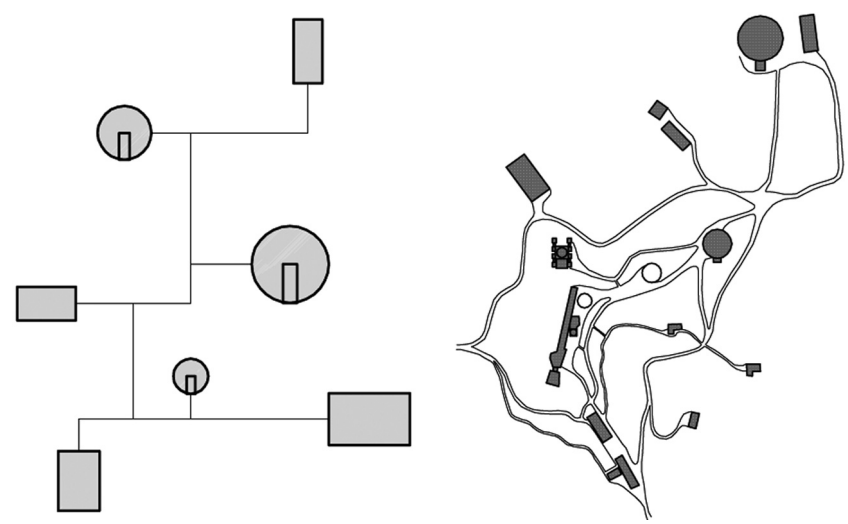

Figura 10. Esquema del tipo disgregado. Planta del Observatorio Mount Wilson como ejemplo.

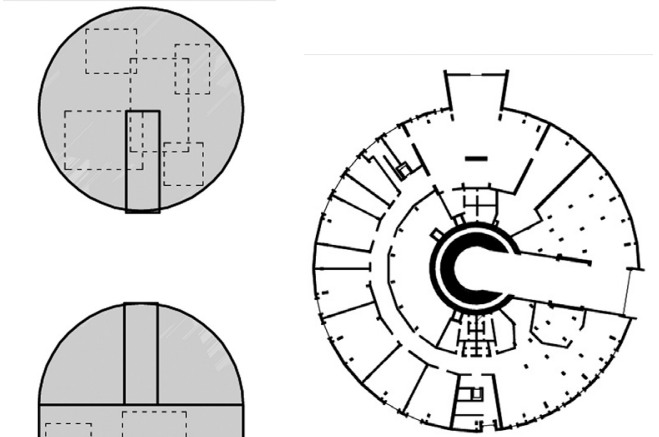

Figura 11. Esquema del tipo compacto en cúpula. Planta del BTA-6 como ejemplo. 


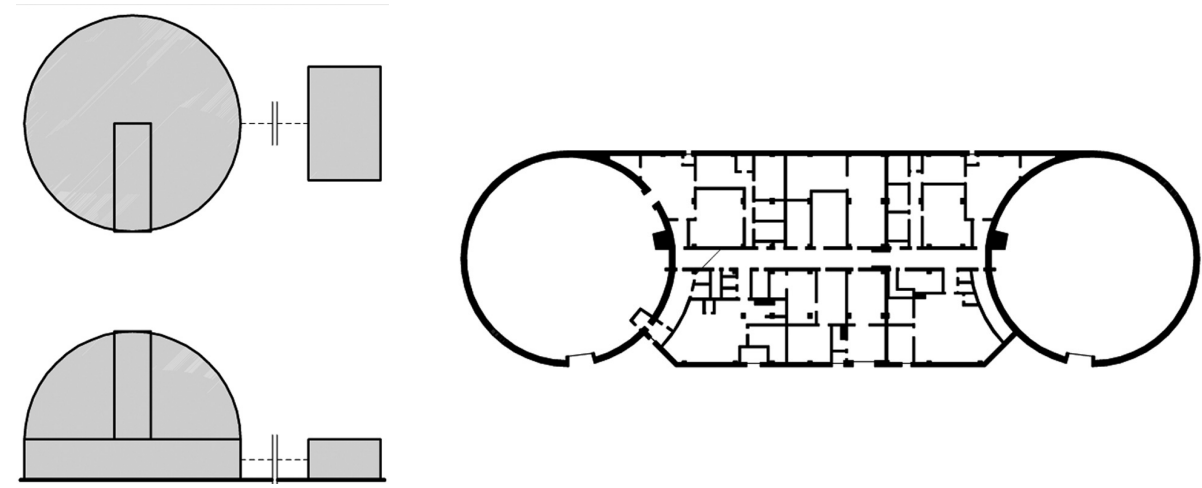

Figura 12. Esquema del tipo de cúpula con anexo. Planta del Observatorio W. M. Keck como ejemplo.

y, a su vez, con el crecimiento de los instrumentos devendrá en el de cúpula sobredimensionada. La búsqueda por evitar las perturbaciones que mermaban los resultados de las observaciones está detrás del origen del tipo disgregado. La liberación de espacio en la base de los reflectores producida por el incremento de tamaño de los mismos da lugar al tipo compacto en cúpula y los avances tecnológicos que posibilitan la separación física entre instrumento astronómico y su operador desembocan en el tipo de cúpula con anexo. Las principales características de estos tipos, la causa subyacente en su origen y sus períodos de vigencia se sintetizan en la tabla de resumen (Tabla 1).

Tabla 1. Los 10 tipos arquitectónicos de observatorio astronómico.

\begin{tabular}{|c|c|c|c|}
\hline Tipo & Periodo & Origen & $\begin{array}{l}\text { Características } \\
\end{array}$ \\
\hline De arco & 1259 - s. XV & $\begin{array}{l}\text { La búsqueda de una mayor } \\
\text { precisión desembocó en } \\
\text { instrumentos tan grandes que } \\
\text { debían ser fijos }\end{array}$ & $\begin{array}{l}\text { - Gran instrumento de arco fijo. } \\
\text { - Alineación norte a sur. } \\
\text { - Algunas funciones astronómicas secundarias incluidas. } \\
\text { - Situación en entorno periurbano. }\end{array}$ \\
\hline Plataforma & s. XV - s. XVII & $\begin{array}{l}\text { Surge para lograr mejores } \\
\text { condiciones de observación con } \\
\text { instrumentos portátiles. }\end{array}$ & $\begin{array}{l}\text { - Superficie abierta de investigación } \\
\text { - A veces incluye un espacio cubierto } \\
\text { - Situación en una posición elevada o adosada a una estructura } \\
\text { existente } \\
\text { - Muy expuesto al clima }\end{array}$ \\
\hline Palaciego & 1576 - s. XVIII & $\begin{array}{l}\text { Es el resultado del intento } \\
\text { de generar una arquitectura } \\
\text { para acomodar la observación } \\
\text { astronómica junto con otras } \\
\text { funciones }\end{array}$ & $\begin{array}{l}\text { - Función astronómica incorporada a una arquitectura residencial o } \\
\text { institucional. } \\
\text { - Algunos usos secundarios astronómicos y no astronómicos } \\
\text { incluidos }\end{array}$ \\
\hline $\begin{array}{l}\text { Sala de } \\
\text { Observación }\end{array}$ & 1675 - s.XVIII & $\begin{array}{l}\text { La creciente importancia del } \\
\text { telescopio da lugar a esta } \\
\text { solución de diseño. }\end{array}$ & $\begin{array}{l}\text { - Espacio especialmente adaptado al uso de largos telescopios portátiles } \\
\text { - Esta sala ocupa una posición elevada en el edificio en el que se } \\
\text { implanta } \\
\text { - Su arquitectura resguarda al observador y al telescopio del clima } \\
\text { - Situado en una posición peri-urbana o no urbana }\end{array}$ \\
\hline De torre & s. XVI - s. XXI & $\begin{array}{l}\text { Es la respuesta al desempeño } \\
\text { de la astronomía en un entorno } \\
\text { urbano. }\end{array}$ & $\begin{array}{l}\text { - Diseñado para evitar obstáculos próximos durante la observación } \\
\text { - La mera altura del edificio conlleva problemas de inestabilidad que } \\
\text { reducen la precisión de las observaciones } \\
\text { - Situación en un entorno urbano }\end{array}$ \\
\hline $\begin{array}{l}\text { De cúpula } \\
\text { Integrada }\end{array}$ & $1785-$ s. XXI & $\begin{array}{l}\text { Es la solución alcanzada para } \\
\text { obtener mejores condiciones de } \\
\text { observación para telescopios. }\end{array}$ & $\begin{array}{l}\text { - Mantiene la función de observación protegida de las condiciones } \\
\text { climáticas } \\
\text { - Cada soporte aislado proporciona una alta estabilidad para su } \\
\text { telescopio } \\
\text { - La cúpula permite apuntar en todas direcciones durante la } \\
\text { observación }\end{array}$ \\
\hline $\begin{array}{l}\text { De cúpula } \\
\text { sobredimensionada }\end{array}$ & 1881 - s. XX & $\begin{array}{l}\text { Aparece por el recurrente } \\
\text { crecimiento del telescopio. }\end{array}$ & $\begin{array}{l}\text { - El tamaño de la gran cúpula interfiere con otras funciones } \\
\text { astronómicas } \\
\text { - La cúpula principal está desplazada a un extremo de la planta. }\end{array}$ \\
\hline Disgregado & 1881 - s. XXI & $\begin{array}{l}\text { Es el resultado de las } \\
\text { incompatibilidades y las } \\
\text { diferentes necesidades de } \\
\text { las diversas funciones que } \\
\text { constituyen el observatorio. }\end{array}$ & $\begin{array}{l}\text { - Separación de las diferentes funciones en edificios independientes } \\
\text { - Cada construcción se adapta a las necesidades del uso que contiene } \\
\text { - Distribución de edificios por el terreno } \\
\text { - Situación en un entorno no urbano }\end{array}$ \\
\hline $\begin{array}{l}\text { Compacto en } \\
\text { cúpula }\end{array}$ & 1948 - s. XXI & $\begin{array}{l}\text { La gran cantidad de espacio } \\
\text { liberado por el aumento en la } \\
\text { escala de los telescopios provoca } \\
\text { esta reunificación de funciones }\end{array}$ & $\begin{array}{l}\text { - Todas las funciones agrupadas bajo el telescopio en el edificio de la } \\
\text { cúpula. } \\
\text { - Arquitectura no adaptada a funciones secundarias. } \\
\text { - Situado en un entorno no urbano. }\end{array}$ \\
\hline Cúpula con anexo & 1993 - s. XXI & $\begin{array}{l}\text { Esta solución es el resultado } \\
\text { del desarrollo tecnológico } \\
\text { de la informática y las } \\
\text { telecomunicaciones. }\end{array}$ & $\begin{array}{l}\text { - Posible separación física del telescopio y el resto de funciones } \\
\text { - Relación virtual entre el observador y el telescopio } \\
\text { - Tanto la cúpula como el edificio secundario pueden especializarse } \\
\text { para las funciones que contienen } \\
\text { - Situación en un entorno no urbano }\end{array}$ \\
\hline
\end{tabular}




\section{CONCLUSIÓN}

Aunque algunos de los 10 tipos propuestos han quedado obsoletos (de arco, plataforma, palaciego y de sala de observación) y un diseño en base a sus características carecería de sentido en el presente, el imprevisible desarrollo que puedan presentar la astronomía o la innovación tecnológica incluso podría devolverles una improbable vigencia. Por otro lado, no existe un único tipo válido para el observatorio actual ya que cada opción se puede adecuar mejor a las circunstancias que definen un determinado centro astronómico. Su configuración dependerá del grado de profesionalización, de la ubicación, del tamaño de los instrumentos, del programa de usos, etcétera.

Con las nuevas tendencias proyectuales de la actualidad, los observatorios astronómicos que se alejan de la repetición de los patrones consolidados y cuyos diseños se basan en el encaje de los requisitos científico-técnicos con los criterios propios de la arquitectura contemporánea son poco frecuentes. Cuando esta particularidad se produce, los resultados se alejan tanto de los cánones habituales que hasta cuesta identificar esos edificios como observatorios. Sin embargo, los resultados son normalmente asimilables a alguno de los tipos referidos.

En todo caso, el análisis tipológico del observatorio constituye una herramienta para su estudio histórico y para una mejor comprensión de su condición. Esto posibilita una comprensión profunda sobre su desarrollo y sobre los atributos que lo definen, lo que, desconociendo cómo será su evolución venidera, será de utilidad a la hora de afrontar el proyecto de un futuro observatorio, independiente de su escala, presupuesto o profesionalización.

Aun así, la evolución de los observatorios ha estado fuertemente marcada por los avances técnicos y por el progreso astronómico, por lo que tanto el desarrollo tecnológico como los nuevos descubrimientos de la ciencia puedan producir nuevas condiciones o requerir diferentes soluciones que alteren los tipos aquí expuestos o, incluso, que generen otros nuevos.

\section{REFERENCIAS}

(1) Brown, D. (2016). An introductory view on archaeoastronomy. Journal of Physics: Conference Series, 685: 1-16. https:// doi.org/10.1088/1742-6596/685/1/012001

(2) Castro Tirado, M.A. (2019). Astronomical Observatories: from the prehistory to the XVIIIth century. Revista Mexicana Astrofísica. Conference Series, 51: 1-8. https://doi.org/10.22201/ia.14052059p.2019.51.02

(3) George, A. (2005). The Tower of Babel: Archaeology, history and cuneiform texts. Archiv für Orientforschung, 51: 75-95.

(4) Mundt, C.S. (1927). Notable astronomers of past ages. II. - Ptolemy. Publications of the Astronomical Society of the Pacific 39(228): 122-126. https://doi.org/10.1086/123689

(5) Gargatagli, M. (1999). La historia de la escuela de traductores de Toledo. Quaderns. Revista de treducció, 4: 9-13. Recuperado de https://www.raco.cat/index.php/QuadernsTraduccio/article/view/25206

(6) Sayili, A. (1960). The Observatory in Islam and its place in the general history of astronomy, p. 4, Ankara: Türk Tarih Kurumu Basimevi.

(7) Dreyer, J.L.E. (1890). Tycho Brahe, p. 4-5, Edimburgo: Adam and Charles Black.

(8) Gunther, R.T. (1932). Early Science in Oxford, p. 71, Londres: Dawsons of Pall Mall.

(9) Zinner, E. (1943). Entstehung und Ausbreitung der Coppernicanischen Lehre, p. 408-417, Erlangen: Kommissionsverlag von Max Mencke.

(10) Todd, D. (1922). Astronomy: the science of the heavenly bodies, p. 4, Londres: Harper \& Brothers.

(11) Pannekoek, A. (1961). A history of astronomy, p.208, Nueva York: Dover.

(12) Gaulke, K. (2009). The first European observatory o the sixteenth century, as founded by Landgrave Wilhelm IV of Hesse-Kassel: a serious historiographic category or a misleading marketing device. En: Strano, G. (Ed.) European Collection of Scientific Instruments, 1550-1750 (pp.87-100). Boston: Brill.

(13) Zinner, E. (1956). Astronomische Instrumente des 11. Bis 18. Jahrhunderts, p. 221, Munich: CH Beck.

(14) Donnelly, M. C. (1971). A short history of observatories, p. 4-5, Oregon: University of Oregon Books.

(15) Perrault, C. (1909). Memories de ma vie, p. 219-221, París: Bonefon.

(16) Wolf, C. (1902). Histoire de l'Observatoire de Paris, p. 396-415, París: Gauthier-Villars Imprimeur-Libraire.

(17) Forbes, E. (1975). The Greenwich Observatory origins and early development. Nature, 255: 587-592. https://doi. org/10.1038/255587ao

(18) Chapman, A. (2013). Thomas Hornsby and the Radcliffe Observatory. En Fauvel, J. (Ed.) Oxford figures, Eight Centuries of the Mathematical Sciences (pp. 202-220). Oxford: Oxford University Press.

(19) Ussher, H. (1787). Account of the Observatory Belonging to Trinity College, Dublin. The Transactions of the Royal Irish Academy, 1: 3-21. Recuperado de https://www.jstor.org/stable/30079201

(20) Smiley, C. H. (1938). The History of the Lick Observatory. The Scientific Monthly, 47(2): 128-135.

(21) Misch, A., Stone, R. (1998). Building the Observatory. Web of The Lick Observatory Historical Collections. http://collections.ucolick.org/archives_on_line/

(22) Holden, E.S. (1891). The Observatory of Nice. Astronomical Society of the Pacific, 3(15): 123-125. https://doi. org/10.1086/120269

(23) A History of Palomar Observatory (2019). http://www.astro.caltech.edu/palomar/about/history.html

(24) The 200-inch (5.1-meter) Hale Telescope (2019). http://www.astro.caltech.edu/palomar/about/telescopes/hale.html

(25) Leverington, D. (2017). Observatories and Telescopes of Modern Times, p. 107, Cambridge: Cambridge University Press. 
(26) Finn, R. (1985). The W. M. Keck Observatory. Engineering and Science, 48(3): 5-10.

(27) Moneo, R. (1978). On Typology. Oppositions, 13: 188-211.

(28) Quatremère de Quincy, A. C. (1832). Dictionnaire historique de l'architecture, p. 629-631, París: Librairie D’Adrien Le Clere et Cie.

(29) Moneo, R. (2004). Sobre la noción de tipo. En Marques, F. (Ed.) Rafael Moneo. 1967-2004 (pp.584-607). Madrid: El Croquis Editorial.

(30) Argan, G.C. (1973). El concepto del espacio arquitectónico desde el Barroco a nuestros días. p. 30-34, Buenos Aires: Nueva visión.

(31) Martín Hernandez, M.J. (1984). La tipología en arquitectura (Tesis doctoral no publicada). Las Palmas: Universidad de Las Palmas de Gran Canaria. Recuperado de https://accedacris.ulpgc.es/bitstream/10553/1914/1/779.pdf

(32) Maure, L. (2009). La superación del concepto de ‘tipo’ en la arquitectura contemporánea. Cuaderno de Notas, 12 : 1-8. Recuperado de http://oa.upm.es/4469/

(33) Müller, P. (1978). Sternwarten in Bildern: Architektur und Geschichte der Sternwarten von den Anfängen bis ca. 1950, p. 252-257, Berlin: Springer-Verlag.

(34) Waumans, A.A. (2013). The Typology of Astronomical Observatories (Tesis doctoral no publicada). Delft: Delft University of Technology. 